\title{
Histological and Immunohistological Evaluation of the Tissue Response of a New Barrier Material Based on D,L-Polylactide, Trimethylene Carbonate and Caprolactone to Prevent Peritoneal Adhesion Formation
}

Constanze NE Planck ${ }^{1,5}$, Volker H Schmitt ${ }^{2}$, Bernhard Krämer ${ }^{1}$, Andreas Mamilos ${ }^{2}$, Taufiek K Rajab ${ }^{3}$, David Hollemann ${ }^{2}$, Christian Wallwiener ${ }^{1}$, Helmut Hierlemann $^{4,5}$, Heinrich Planck ${ }^{4,5}, \mathrm{C}$ James Kirkpatrick ${ }^{2}$ and Christoph Brochhausen ${ }^{2^{*}}$

${ }^{1}$ Department of Gynecology and Obstetrics, Eberhard Karls University, Tuebingen, Germany

${ }^{2}$ REPAIR-lab, Institute of Pathology, Johannes Gutenberg-University, Mainz, Germany

${ }^{3}$ Brigham and Women's Hospital, Harvard Medical School, Boston, USA

${ }^{4}$ Institute of Textile Technology and Process Engineering, Denkendorf, Germany

${ }^{5}$ German Centre of Biomaterials and Artificial Organs e.V. Denkendorf, Germany

"Corresponding author: Christoph Brochhausen, REPAIR-lab, Institute of Pathology, University Medical Center, Johannes Gutenberg University, Mainz, Germany, Tel: ++49 061311773 07; Fax: ++49 06131174773 07; E-mail: brochhausen@pathologie.klinik.uni-mainz.de

Rec date: March 07, 2014, Acc date: May 20 2014, Pub date: May 23, 2014

Copyright: (c) 2014 Planck CNE, et al. This is an open-access article distributed under the terms of the Creative Commons Attribution License, which permits unrestricted use, distribution, and reproduction in any medium, provided the original author and source are credited.

\begin{abstract}
Serosal membranes cover body cavities and guarantee frictionless gliding of inner organs. Serosal damage carries the risk of adhesion formation, which represents a relevant postoperative complication. Due to pathophysiological similarities between serous and dermal wound healing, the efficacy of SupraSeal ${ }^{\circledR}$ in intraabdominal adhesion prevention was investigated at early time points. After standardized serosal damage, Wistar rats were examined macroscopically, histologically and immunohistochemically on days two, four and eight postoperatively. On day eight, all specimens of the control group presented distinctive adhesions consisting of fibrous tissue bands. In contrast, most specimens covered by SupraSeal ${ }^{\circledR}$ were adhesion-free. Merely mild adhesion formation due to suture material used for fixation was detected. Histologically, SupraSeal ${ }^{\circledR}$ revealed rapid clearance of fibrin and a marked lack of fibrosis. For the first time, the histomorphological effects of SupraSeal ${ }^{\circledR}$ on adhesion formation over time are described and the relevance of the early stage of wound healing elucidated.
\end{abstract}

Keywords: Adhesion prevention; Intraperitoneal adhesions; Postoperative adhesion formation; Barrier material; Barrier membrane

\section{Introduction}

Intraperitoneal adhesions are bands of connective tissue which originate from the peritoneum after serosal damage due to trauma, surgical intervention and inflammation [1]. A multistep process lead to the development of this organised tissue with collagen fibre deposition, vascular and nerve fibre in growth. These tissue bands cause adherence between intraperitoneal organs or between the viscera and the abdominal wall [2,3]. Clinically, postoperative adhesion formation represents a relevant problem since they present a common complication in gynaecological and abdominal surgery [3-5]. Secondlook analysis revealed that up to $90 \%$ of patients develop adhesions after gynaecological surgery [6,7]. Peritoneal adhesions cause clinically relevant symptoms such as chronic pain, bowel obstruction or infertility $[8,9]$. Furthermore, already existing adhesions lead to prolonged operation times as well as increased rates of surgical complications, including bleeding and organ damage. Consequently, adhesiolysis has become a frequent surgical treatment $[10,11]$. In addition, adhesion formation plays an important economic role, since their annual treatment amounts to $\$ 1.3$ billion in the USA alone [7,9]. Despite the developments during the last few years in reducing trauma by minimally invasive surgical procedures, postoperative adhesions are still responsible for increased postoperative morbidity and thus they still represent a significant problem in interventional medicine [10-13].
Although the formal pathogenesis of adhesion formation has not yet been completely clarified, preliminary findings give insight into the pathophysiological processes. Peritoneal wounds have shown to be completely covered with mesothelial cells within five to ten days independent of the size of the damaged surface [14-17]. Regarding adhesion formation, it was demonstrated that rapid remesothelialization is essential for reducing adhesion formation [18]. Experimental studies revealed that adhesiogenesis results from a cascade of mechanisms in which the mesothelium of the peritoneal serosa, the endothelium, inflammatory cells, fibroblasts and multiple cytokines as well as growth factors and signalling molecules are in an complex interplay [2,3,12]. A prerequisite for peritoneal adhesion formation is mesothelial cell damage with subsequent inflammation $[2,10]$. This inflammatory reaction is controlled by various mediators and causes an elevated vascular permeability, fibrin exudation and the migration of inflammatory cells $[3,19,20]$. Under physiological conditions, the fibrinolytic activity of the peritoneum would lead to dissolution of the fibrin matrix [2]. However, ischaemia, a reduction in fibrinolytic activity by loss of mesothelial cells and the effect of cytokines, disturb the fibrinolytic balance in favour of fibrin formation [21-23]. Fibrin bands, which connect traumatised and surrounding tissue areas, are hence not dissolved and fibroblasts emerge to remodel these fibrin bridges to permanent, well-organized connective tissuecontaining blood vessels and nerve fibres $[3,24,25]$.

Since peritoneal damage with mesothelial-free areas is crucial for the formation of peritoneal adhesions, various material-based approaches are already clinically available to cover and shield denuded 
Citation: Planck CNE, Schmitt VH, Krämer B, Mamilos A, Rajab TK, et al. (2014) Histological and Immunohistological Evaluation of the Tissue Response of a New Barrier Material Based on D,L-Polylactide, Trimethylene Carbonate and Caprolactone to Prevent Peritoneal Adhesion Formation. J Tissue Sci Eng 5: 138. doi:10.4172/2157-7552.1000138

Page 2 of 9

serous surfaces. Besides the anti-adhesive effect, an optimal adhesion barrier should be absorbable and easy to handle also in minimalinvasive surgery and should give good biocompatibility. Despite a variety of liquid, gelatinous and solid barriers in clinical use, the ideal barrier has not yet been found $[3,26]$. In this context, SupraSeal is a biocompatible and degradable membrane which was developed by the Institute of Textile and Process Engineering in Denkendorf (Germany) and is already a promising device in clinical use as a dermal wound dressing under the trade name Suprathel ${ }^{\circ}$ [27]. It is composed of D,Lpolylactide, trimethylene carbonate and caprolactone. These ingredients are already commonly used in surgery in resorbable suture materials as well as osteosynthesis products and the material is entirely degraded into its non-toxic components $[8,14]$. The membrane has a pore size of $2-5 \mu \mathrm{m}$ and a thickness of $70-150 \mu \mathrm{m}$ [27]. Due to the small pore size it is not possible for cells to penetrate the membrane, but a transfer of metabolites between the wound and the surrounding environment is possible $[27,28]$. This membrane shows high plastic deformability at body temperature and can therefore easily adjust to the wound surface. Simultaneously, the glass transition temperature of $32{ }^{\circ} \mathrm{C}$ enables cutting of the membrane at room temperature. In vitro studies have shown that the elongation at break is $250 \%$ whereas the hydrolytical degradation takes five to six weeks [27]. This property makes it possible to use the membrane as a superficial adhesion barrier. This material is already on the market as Suprathel and clinically approved in the treatment of dermal wounds, e.g. burn wounds, skin donor sites or split-thickness skin grafts, and has been reported to give good results $[29,30]$. Regarding the positive clinical experience in the treatment of dermal wounds and in view of the similarities between pathophysiological processes in dermal and serous wound healing, this material has already been tested as an adhesion barrier in animal experiments and a human pilot study with macroscopically promising results $[8,14,28]$. One explanation for the anti-adhesive effect could be swift remesothelialization after treatment with SupraSeal. This was demonstrated in an ultrastructural study comparing various barrier materials to analyze the amount of the remesothelialized surface after peritoneal trauma [18,31]. Furthermore, wound healing-supporting characteristics of this material were considered as a potential reason [27]. However, this membrane is neither commercially available nor yet approved as an adhesion barrier device. Thus, there is a lack of information regarding the histomorphological reaction of this barrier material when used in the peritoneal cavity. Furthermore, after a review of the literature, there is scant information regarding the response of the serosal tissue over time in combination with such a barrier material. This is of special interest, since the early response of barrier materials seems to be crucial for their clinical effects $[3,20]$.

The aim of the present study was to investigate via histological and immunohistological methods the response of the early histomorphological tissue reaction of SupraSeal over time within the treatment of peritoneal lesions. Special interest was given to the elemental processes in adhesion formation such as fibrin exudation, the infiltration of granulocytes, lymphocytes and macrophages and a potential foreign body reaction.

\section{Material and Methods}

\section{Study design, surgical procedure and macroscopic evaluation}

The present investigator initiated prospective, randomised animal study was approved by the ethics commission of the Eberhard-Karls-
University of Tuebingen, Germany. The barrier material was provided by the Institute of Textile Technology and Process Engineering Denkendorf, Germany. The surgical interventions were performed by the animal protection representative of the Eberhard-Karls-University of Tuebingen under aseptic conditions. Anaesthesia was achieved by isoflurane, ketamine and intraperitoneally injected xylacine. A schematic depiction of the experimental procedure is given in Figure 1.

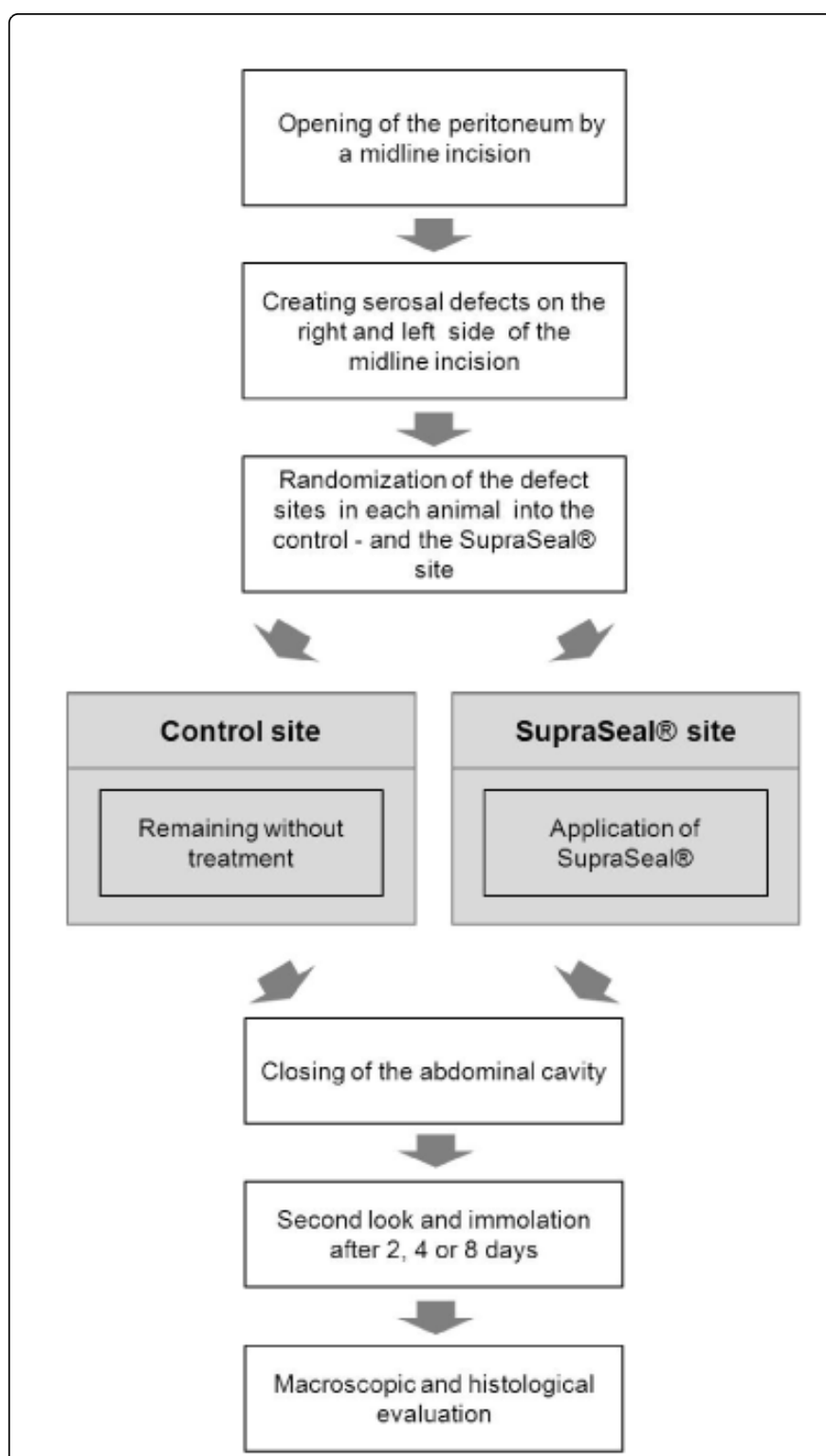

Figure 1: Schematic depiction of the work-flow of the experimental procedure

In detail, 20 female Wistar rats (Charles River, Sulzfeld, Germany) with a weight range from $180 \mathrm{~g}$ to $300 \mathrm{~g}$ were used for this study. After opening the peritoneal cavity by a midline incision of $5 \mathrm{~cm}$, a defect of the serous membrane was created on both sides of the abdominal cavity. As adhesion induction model electrocoagulation and ischaemic sutures were used in Wistar rats, methods which have previously been shown to be reliable [9,32-34]. For this purpose, at the level of the 
Citation: Planck CNE, Schmitt VH, Krämer B, Mamilos A, Rajab TK, et al. (2014) Histological and Immunohistological Evaluation of the Tissue Response of a New Barrier Material Based on D,L-Polylactide, Trimethylene Carbonate and Caprolactone to Prevent Peritoneal Adhesion Formation. J Tissue Sci Eng 5: 138. doi:10.4172/2157-7552.1000138

Page 3 of 9

second mammary gland at a distance of $1.5 \mathrm{~cm}$ from the median incision line, an area of $1.5 \times 1.5 \mathrm{~cm}$ of the peritoneum was defined by four incisions. Afterwards, the peritoneum of this area was removed using forceps. Two incisions then followed, one caudal and one cranial on the outer rim of the defect, to induce bleeding. Additionally, via single sutures ischaemia was created using material that is common in clinical daily routine (Vicryl ${ }^{\circ}$-suture USP 3/0, Ethicon, Somerville, USA). The freestanding ends of the suture material were cut off at a distance of $0.5 \mathrm{~cm}$ from the knot. After peritoneal damage, SupraSeal ${ }^{\circ}$ was implemented on one side of the abdominal wall to cover the lesions. The barrier was therefore cut to a size of $2.5 \mathrm{cmx} 2.5 \mathrm{~cm}$, placed over the wounded peritoneal area and secured on each edge via a single suture. The opposite side of the ventral wall remained uncovered as control. To imitate the fixation sutures applied on the treated abdominal wall, four single sutures without a barrier were placed in four corners of the defect in the control side. Hence, except for the material implementation itself, both sides of the ventral wall in each animal were treated and wounded equally and contained the same amount of suture material. Finally, the abdominal cavity was closed continuously layer for layer. Over two days the rats were treated with the analgesic buprenorphine (0.05 - $0.07 \mathrm{mg} / \mathrm{kg}$ body weight) and controlled four times a day for healing disturbances. The various steps of the surgical procedure are illustrated in Figure 2 by an intraoperative photo-documentation.

Animals were sacrificed after two $(n=7)$, four $(n=6)$ and eight $(n=7)$ days using $\mathrm{CO}_{2}$, followed by second look laparotomy and macroscopic assessment of the extent of adhesion formation. Subsequently, the damaged areas were explanted and fixed in buffered formalin (4\%).

Macroscopically, adhesion formation was evaluated according to the extent of adhesion formation. For this, four categories with a corresponding point value were used: If no adhesions were present, the sample was scored as 0 . Mild adhesion formation, which implied the attendance of one thin, transparent peritoneal adhesion, was scored as 1. Several thin, transparent adhesions or one opaque, non-transparent adhesion were evaluated as moderate postoperative adhesion formation and scored as 2 points. Peritoneal adhesion formation was considered severe when several opaque, non-transparent adhesions or at least one opaque adhesion, which affected abdominal organs other than the intestine, were seen. This scenario was assessed with a point value of 3 . Once each sample of a group (namely both the control and the barrier group on day two, four and eight) was assessed, the average value of this group was determined. According to the average value, the extent of postoperative adhesion formation in each group could be identified. An average score of 0-0.49 points correlated with no adhesion formation, an average of 0.49-1.49 corresponded to mild adhesion formation, a point value of 1.5-2.49 matched with a moderate extent of adhesions and an average score of 2.5 and higher described severe adhesion formation (Table 1).

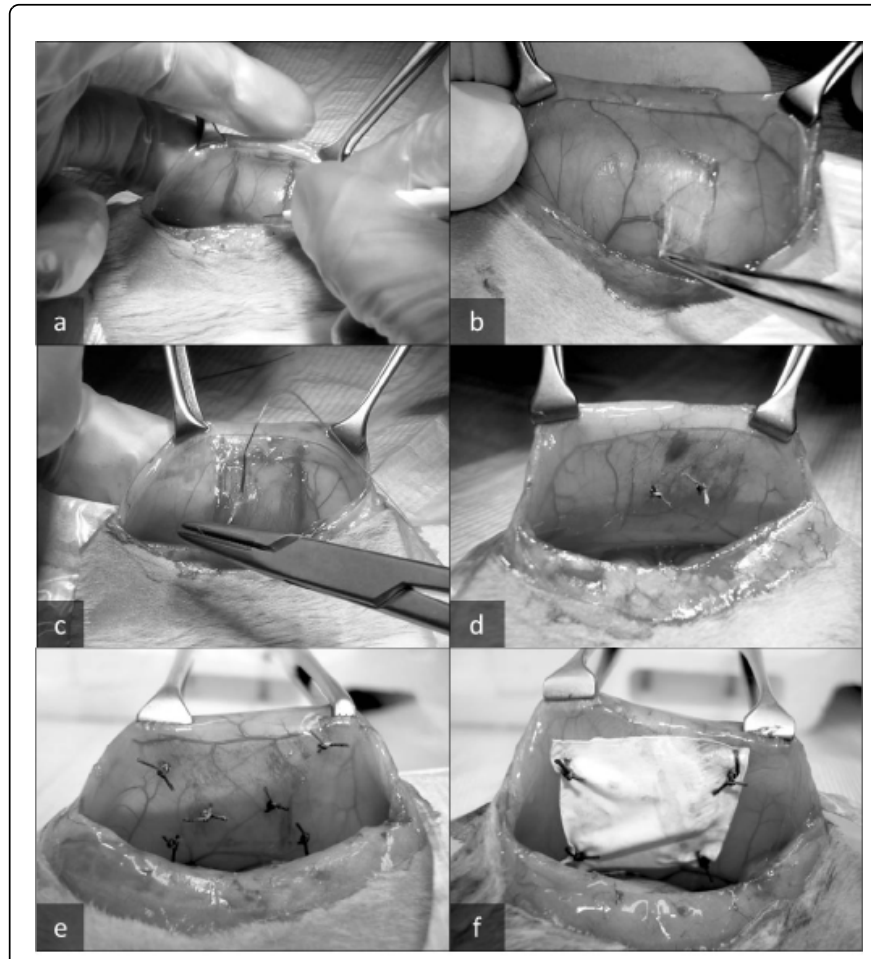

Figure 2: Macroscopic photographs from the different steps of the surgical procedure: After opening the abdominal cavity via midline incision the defect areas of the abdominal wall were defined and marked using a scalpel on both sidewalls of the peritoneum (a). The parietal peritoneum of the circumscribed areas was then removed with a forceps (b) and ischaemic buttons were created using two single sutures $(c+d)$. Since the suture material induces adhesion formation, four additional single sutures were used to imitate the fixation of a barrier material on the control abdominal sidewalls (e). At the barrier-treated ventral walls, the damaged areas were covered by SupraSeal ${ }^{\star}$ which was fixed by four single sutures (f).

\begin{tabular}{|c|c|c|c|c|}
\hline \multicolumn{5}{|c|}{ Macroscopic evaluation of the extent of postoperative adhesion formation } \\
\hline point value & 0 & 1 & 2 & 3 \\
\hline average score & $0-0.49$ & $0.5-1.49$ & $1.5-2.49$ & from 2.5 \\
\hline definition & none & Mild & moderate & Severe \\
\hline criteria & no adhesions & one thin, transparent adhesion & $\begin{array}{l}\text { several thin, transparent } \\
\text { adhesions or one opaque, non- } \\
\text { transparent adhesion }\end{array}$ & $\begin{array}{l}\text { several opaque, non- } \\
\text { transparent adhesions or at } \\
\text { least one opaque adhesion to } \\
\text { abdominal organs other than } \\
\text { the intestine }\end{array}$ \\
\hline
\end{tabular}

Table 1: The evaluation score used to assess the macroscopical extension of postoperative adhesion formation including the interpretation of the average score. 
Citation: Planck CNE, Schmitt VH, Krämer B, Mamilos A, Rajab TK, et al. (2014) Histological and Immunohistological Evaluation of the Tissue Response of a New Barrier Material Based on D,L-Polylactide, Trimethylene Carbonate and Caprolactone to Prevent Peritoneal Adhesion Formation. J Tissue Sci Eng 5: 138. doi:10.4172/2157-7552.1000138

Page 4 of 9

\section{Histological as well as immunohistological staining and evaluation}

For histological evaluation and immunohistochemical detection of macrophages, the specimens were dehydrated in standardised, automated processes and embedded in paraffin. From the paraffin blocks slices of $4 \mu \mathrm{m}$ thickness were cut and then stained with haematoxylin-eosin. The development of fibrosis was demonstrated by elastica van Gieson staining. To distinguish granulocytes from lymphocytes within the inflammatory infiltrates chloracetate-esterase staining was performed. Macrophages were immunostained with monoclonal antibodies against CD68 (1:600 Dako, Hamburg, Germany) using the automated standardized peroxidaseantiperoxidase method in a Dako Autostainer Plus (Dako, Hamburg, Germany). All staining reactions were performed under standardized conditions.

The specimens were evaluated semi-quantitatively by two independent investigators with a view to the inflammatory reaction given by the the amount of inflammatory cells, namely granulocytes and lymphocytes or plasma cells. In addition, the amount of CD68positive macrophages and the foreign body reaction given by the density of multinucleated foreign body giant cells were assessed. Furthermore, the accumulation of fibrin was estimated and fibrosis was evaluated by the extent of fibrotic bands using the categories no fibrosis, narrow band, moderate band, broad band and extensive band.

The score used to evaluate the tissue response is shown in Table 2. In order to compare the results of both groups, the average value was calculated for each variable at each time point and interpreted according to the score.

\begin{tabular}{|l|l|l|l|l|l|}
\hline \multicolumn{7}{|c|}{ Histological evaluation of the tissue response } \\
\hline point value & 0 & 1 & 2 & 3 & 4 \\
\hline average score & $0-0.49$ & $0.5-1.49$ & $1.5-2.49$ & $2.5-3.49$ & from 3.5 \\
\hline inflammation & none & mild & moderate & severe & \\
\hline fibrosis & none & $\begin{array}{l}\text { narrow } \\
\text { band }\end{array}$ & $\begin{array}{l}\text { moderate } \\
\text { band }\end{array}$ & wide band & $\begin{array}{l}\text { extensive } \\
\text { band }\end{array}$ \\
\hline macrophages & none & mild & moderate & severe & \\
\hline $\begin{array}{l}\text { foreign body } \\
\text { giant cells }\end{array}$ & none & mild & moderate & severe & \\
\hline fibrin & none & little fibrin & $\begin{array}{l}\text { moderate } \\
\text { amount }\end{array}$ & $\begin{array}{l}\text { large } \\
\text { amount }\end{array}$ & \\
\hline
\end{tabular}

Table 2: The evaluation score used to estimate the tissue response of each sample including the interpretation of the average score.

\section{Statistical analysis}

The data acquisition and statistical evaluation including the comparison of the groups occurred via Excel 2010 (Microsoft, Redmond, WA, USA) and SPSS 20 (IBM, Armonk, NY, USA) using two-way analysis of variance with repeated measurements at a statistical significance level of $\mathrm{p}<0.05$.

\section{Results}

\section{Macroscopic evaluation}

At all analysed time-points in all animals, the damaged areas were easily distinguishable from undamaged peritoneum, which macroscopically revealed a filmy thin layer with a shining opaque surface. SupraSeal ${ }^{\circ}$ was visible at all time points at the treated sites and showed a firm, non-deformable consistency when the samples were explanted. The material was still located at the implanted areas, but in some cases it was sheared off one of the fixation sutures. Nevertheless, the barrier was still firmly fixed by the remaining stitches. No correlation between the level of fixation and the formation of peritoneal adhesions was detected. All adhesions were evaluated according to the scoring system illustrated in Table 1.

After two days, adhesion formation had already occurred but, compared to the samples on the following days, these adhesions were overall loose, predominantly transparent and less solid. In the control group, adhesions between the serosal wound and the mesenterial fat tissue as well as the small and the large bowel had been formed in the entire area of the lesions. Only one specimen of the control was completely free of adhesions. The only adhesion formation that was detectable on day two originated from the suture material which was used to fix the barrier to the wound surface. On average, the extent of adhesion formation in the control was moderate and therefore significantly higher compared to the barrier sides where no adhesions could be found.

On day four, the adhesions were stronger and more solid compared to day two. In the control, adhesion formation remained at a moderate level and adhesions were more distinctive. On the barrier side, also at day four only for two specimens could mild adhesions be found originating from the barrier material. Hence, regarding the macroscopic scoring, there was a significant difference between the treated and untreated ventral wall sides also on day four. Four specimens of the barrier side as well as one sample of the control were completely free of adhesions. Furthermore, in two specimens of the control and one specimen of the barrier group, superficial fibrosis in the area of the lesion was present, macroscopically seen as greyishwhite deposits on the surface.

Eight days postoperatively, the samples of the control revealed severe adhesion formation which was characterized by strongly formed broad adhesive bands. On the treated sides, i.e. with the barrier, moderate adhesions were present originating from the suture material but only slight adhesion formation was found on the barrier itself in some samples. Furthermore, superficial fibrous tissue at the surface without the formation of adhesive bands to the abdominal wall was seen in three specimens of the control as well as in two barrier samples.

The results of the macroscopic evaluation are summarized in Table 3. Illustrations of an adhesion free ventral wall treated with SupraSeal ${ }^{\circ}$ (a) compared to extensive adhesion formation at a control side (b) after eight days are given in Figure 3. 
Citation: Planck CNE, Schmitt VH, Krämer B, Mamilos A, Rajab TK, et al. (2014) Histological and Immunohistological Evaluation of the Tissue Response of a New Barrier Material Based on D,L-Polylactide, Trimethylene Carbonate and Caprolactone to Prevent Peritoneal Adhesion Formation. J Tissue Sci Eng 5: 138. doi:10.4172/2157-7552.1000138

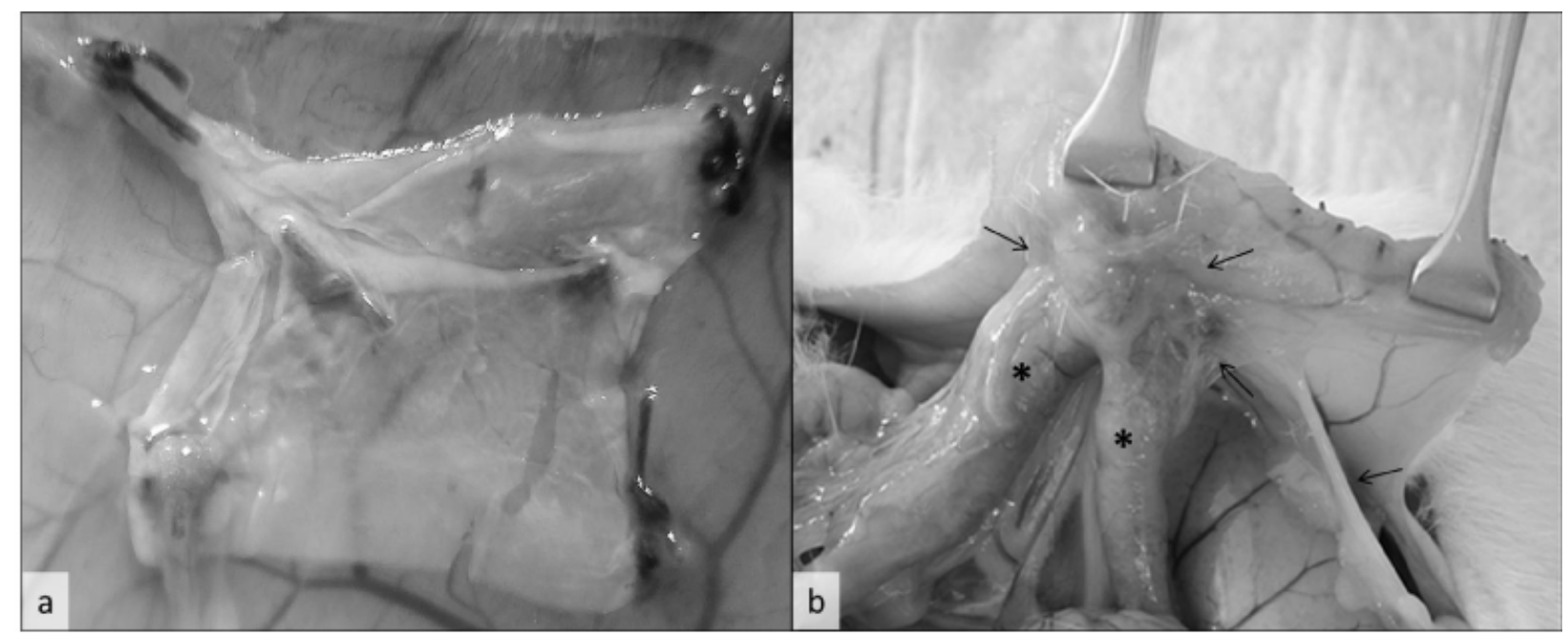

Figure 3: The abdominal cavity after eight days with an adhesion-free sidewall treated with the barrier (a) and extensive adhesion formation between the abdominal wall and the bowel $\left({ }^{*}\right)$ in the entire area of the lesion (arrow) at an untreated ventral wall (b).

\begin{tabular}{|c|l|c|l|l|}
\hline \multicolumn{2}{|c|}{ second look } & \multicolumn{1}{c|}{2 days } & \multicolumn{1}{c|}{ 4 days } & 8 days \\
\hline \multirow{2}{*}{ group } & control & mild $(1.86)$ & moderate $(1.83)$ & severe $(2.71)$ \\
\cline { 2 - 5 } & SupraSeal ${ }^{\circledR}$ & none $(0.14)$ & none $(0.33)$ & moderate $(2.00)$ \\
\hline
\end{tabular}

Table 3: Summary of the macroscopic adhesion scoring after two, four and eight days postoperatively. The average value is given in brackets

\section{Histological evaluation}

Detection of Fibrin over time: After two days, in both groups small amounts of fibrin were detectable with a higher level in the treated sides compared to the control samples. In the control group, adhesions between the parietal peritoneum and the mesenteric fat tissue were evident in the areas of fibrin deposition (Figure 4a). At day four and day eight, these adhesions became more firm and organised by fibrous and fat tissue (Figure $4 \mathrm{~b}$ and $4 \mathrm{c}$ ). Although the average level of fibrin in the barrier samples was two times higher than in the control sides after two days, the specimens treated with the barrier showed a rapid clearance of fibrin with a total absence at day four (Figure 4e). By comparison, minimal amounts of fibrin were present in the control samples four days postoperatively (Figure $4 \mathrm{~b}$ ). It is noteworthy that fibrin in the barrier group was accentuated below the barrier material (Figure 4d). On day eight, neither in the control nor in the barrier samples could any fibrin deposits be found. Whereas large areas of the surface covered by the barrier material were adhesion-free (Figure 4e) at day eight, adhesion formation in these specimens was accentuated at the fixation sites.

Evaluation of the inflammatory response over time: Inflammation, evidenced by granulocytes and lymphocytes or plasma cells was moderate in the control samples at all analysed time points (Figure 5b, $5 e$ and $5 h)$.

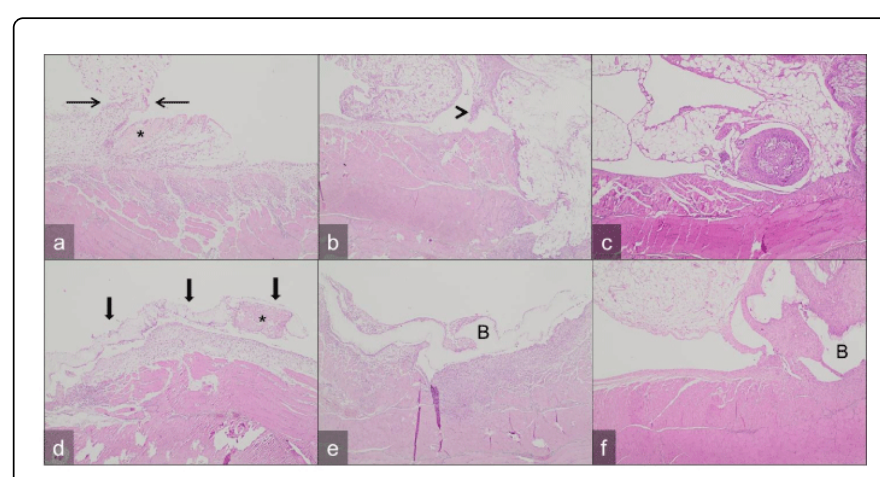

Figure 4: Haematoxylin and eosin staining of the control (a-c) and SupraSeal group (d-f): after two days, adhesion formation and fibrin deposits $\left(^{*}\right)$ were visible in the control group (arrows) (a, $\mathrm{x} 100$ ), whereas in the SupraSeal ${ }^{\circ}$ group the entire surface and fibrin deposits $\left(^{*}\right)$ were covered by the barrier material (broad arrows) without adhesion formation (d, x100). Four days postoperatively there was a moderate formation of adhesions in the control specimens with small fibrin deposits (arrow head) (b, x200). In the SupraSeal samples, the barrier material (B) was covered by almost flat cells with less adhesion formation compared to the control (e, $\mathrm{x} 200$ ). Eight days after surgery, in all specimens of the control as well as in parts of the barrier group adhesions composed of fat and connective tissue were detectable (c, x200; f, x200)

In contrast, the treated ventral sides were associated with a mild inflammatory reaction on days two (Figure 6b) and four (Figure 6e). Eight days after surgery, both groups revealed the same level of inflammatory reaction, which was moderate at this time point (Figure $5 \mathrm{~h}$ and $6 \mathrm{~h}$ ). In both the barrier and the control specimens, a dominance of granulocytes compared to lymphocytes was evident at days two and four. Eight days postoperatively, granulocytes remained dominant in the control, whereas in the barrier samples lymphocytes became prevalent. Overall, in both groups over time a decrease of 
Citation: Planck CNE, Schmitt VH, Krämer B, Mamilos A, Rajab TK, et al. (2014) Histological and Immunohistological Evaluation of the Tissue Response of a New Barrier Material Based on D,L-Polylactide, Trimethylene Carbonate and Caprolactone to Prevent Peritoneal Adhesion Formation. J Tissue Sci Eng 5: 138. doi:10.4172/2157-7552.1000138

Page 6 of 9

granulocytes combined with an increase of lymphocytes was observed (Figure 5b, 5e, 5h, 6b, 6e and 6h).

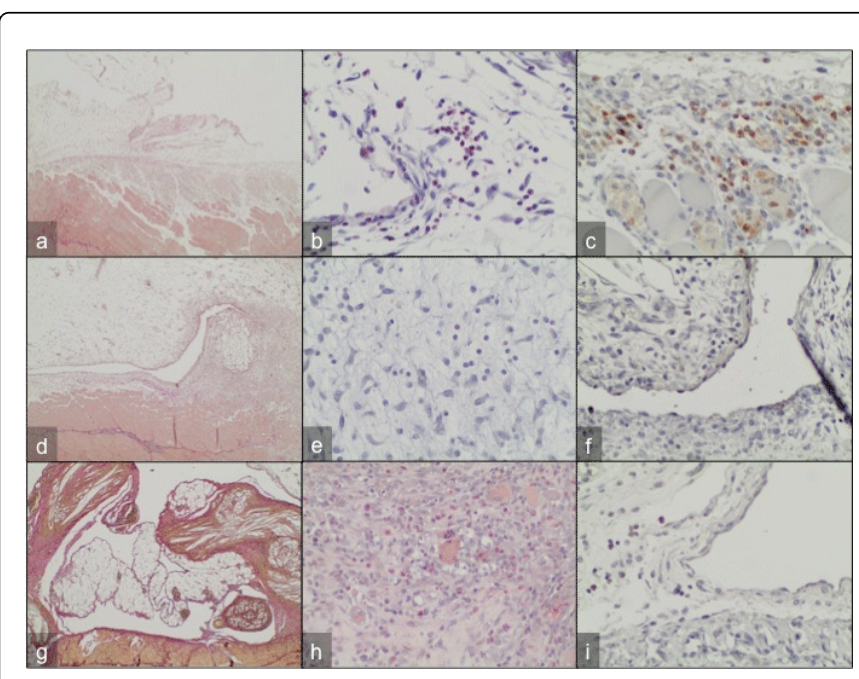

Figure 5: Histological and immunohistochemical staining of control specimens after two (a-c), four (d-f) and eight days (g-i): The EvG stain revealed minimal fibrosis after two days (a, x100). Inflammation was moderate in the ASD stain, which revealed pink coloured granulocytes $(\mathrm{b}, \mathrm{x} 400)$. In addition, a moderate infiltration of brown-stained CD68-positive macrophages was detected (c, $\mathrm{x} 400$ ). Four days postoperatively, fibrosis was still minimal in the control ( $\mathrm{d}, \mathrm{x} 100 \mathrm{EvG})$. The inflammatory response remained moderate (e, ASD, $x 400)$ and a mild macrophage infiltration could be seen ( $f$, anti-CD68 stain, $x 400$ ). Eight days postoperatively, a narrow fibrosis band was visible in the EvG stain ( $\mathrm{g}, \mathrm{x} 200)$ and the ASD stain revealed a moderate inflammatory reaction $(h, x 400)$. A mild infiltration of macrophages was seen via immunohistochemistry (i, anti-CD68 stain, x400).

Immunohistological detection of macrophage infiltration: The infiltration with CD68-positive macrophages was moderate in both groups at day two (Figure $5 \mathrm{c}$ and $6 \mathrm{c}$ ). Four days after treatment, the control specimens presented a mild infiltration of macrophages (Figure 5f), whereas in the barrier samples a moderate reaction was present (Figure 6f). Eight days postoperatively, the infiltration with CD68-positive macrophages was mild in both groups (Figure $5 \mathrm{i}$ and $6 i)$.

Evaluation of the foreign body reaction: No foreign body reaction occurred in the control specimens at any time point. In the barrier samples, no foreign body reaction was present at day two and day eight. Four days postoperatively a mild foreign body reaction was observed in the form of multinucleated giant cells.

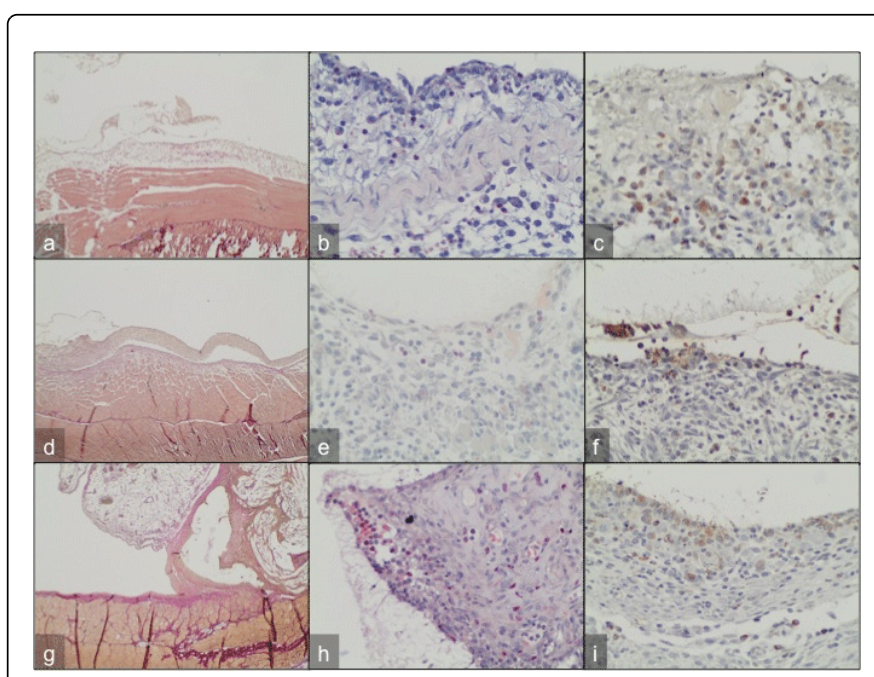

Figure 6: Histological and immunohistochemical staining of SupraSeal ${ }^{\circ}$-treated samples after two (a-c), four (d-f) and eight days (g-i): Minimal fibrosis was seen after two days (a, EvG, x100) and the inflammatory response was mild (b, ASD, x400). Macrophage infiltration was moderate two days postoperatively (c, anti-CD68 stain, $\mathrm{x} 400$ ). After four days, minimal fibrosis was detected in the elastica van Gieson $(\mathrm{EvG})$ stain (d, x100). Inflammation was mild (e, ASD, 400x) and a moderate number of macrophages was present (f, anti-CD68 stain, $\mathrm{x} 400$ ). Eight days after surgery, the fibrosis band was still narrow in the EvG stain (g, x200). A moderate inflammatory reaction could be seen ( $h, A S D, x 400)$ as well as a mild macrophage infiltration (i, anti-CD68 stain, $\mathrm{x} 400$ ).

Evaluation of fibrosis over time: At all time points, the control and the barrier groups demonstrated a narrow fibrotic tissue band with marked differences in the location. Whereas fibrotic bands in control specimens were located between the damaged areas and the neighbouring tissue (Figure 5a, 5d, and $5 \mathrm{~g}$ ), fibrotic bands in the barrier-treated samples were mainly located at the surface of the barrier material (Figure 6a, 6d and 6g). Furthermore, in both analysed groups some flat mesothelial cells could be demonstrated on the surface at all time-points even on day two, indicating the commencement of remesothelialization of the barrier-coated surface.

The histological findings are summarized in Table 4 and a depiction of the results in the course of time is given in Figures 4-6.

\begin{tabular}{|c|c|c|c|c|c|c|}
\hline second look & Group & Inflammation & Macrophages & fibrosis & $\begin{array}{l}\text { foreign body } \\
\text { reaction }\end{array}$ & Fibrin \\
\hline \multirow[t]{2}{*}{2 days } & Control & moderate (1.67) & moderate (1.67) & narrow band $(0.83)$ & none $(0.00)$ & little $(0.67)$ \\
\hline & SupraSeal ${ }^{\circledR}$ & mild (1.33) & moderate (1.83) & narrow band $(0.67)$ & none $(0.00)$ & little (1.33) \\
\hline \multirow[t]{2}{*}{4 days } & Control & Moderate (1.50) & mild $(0.75)$ & narrow band (1.00) & none $(0.00)$ & little $(0.50)$ \\
\hline & SupraSeal ${ }^{\circledR}$ & mild (1.25) & moderate (1.75) & narrow band (1.00) & mild $(0.75)$ & none $(0.00)$ \\
\hline 8 days & control & moderate $(2.14)$ & mild $(0.67)$ & narrow band (1.29) & none $(0.29)$ & none $(0.00)$ \\
\hline
\end{tabular}


Citation: Planck CNE, Schmitt VH, Krämer B, Mamilos A, Rajab TK, et al. (2014) Histological and Immunohistological Evaluation of the Tissue Response of a New Barrier Material Based on D,L-Polylactide, Trimethylene Carbonate and Caprolactone to Prevent Peritoneal Adhesion Formation. J Tissue Sci Eng 5: 138. doi:10.4172/2157-7552.1000138

Page 7 of 9

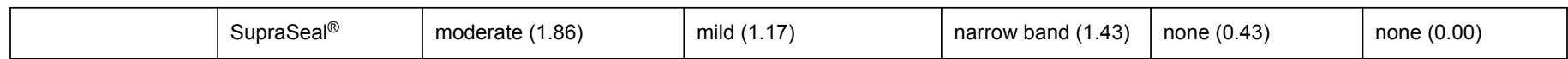

Table 4: Summary of the tissue response after two, four and eight days postoperatively. The average value is given in brackets.

\section{Statistical analysis}

The macroscopic evaluation was significant at all analysed time points $(\mathrm{p}<0.0001)$. Hence, there was a significant difference in the outcome of adhesion formation between the barrier and the control samples. In the histologic examination, no statistical significant differences for the analysed parameters could be found ( $p>0.05)$ except for the migration of macrophages. For this parameter significant differences in the barrier and control group were evident at all analysed time points $(\mathrm{p}<0.008)$. Consequently, regarding the histological evaluation and the macroscopic tissue reaction, the barrier and the control specimens did not differ statistically from each other, except for the infiltration with macrophages.

\section{Discussion}

In the present study, the histomorphological time course of the early tissue reaction to an absorbable barrier membrane based on a copolymer of D,L-polylactide, trimethylene carbonate and caprolactone, was examined in comparison to an untreated control in an animal model of peritoneal adhesion formation. Furthermore, our results revealed a good anti adhesive property of the synthetic membrane.

The macroscopical findings of this study, with adhesion formation to a significantly lesser extent due to the implantation of Supra Seal compared to the untreated control group, are in line with former animal studies from our group [8,9]. However, to our knowledge this is the first animal study to analyze on a histomorphological level the early time course of treatment with a new anti adhesive device. Since peritoneal adhesion formation represents a cascade-like dynamic process with which a potential barrier material interferes, there are two underlying reasons for an analysis at early time points: firstly, the early effects of the material in the wounded area are of interest with respect to the interaction with the adhesion formation cascade [3]. This aspect could give an innovative input for further optimization of barrier materials [3]. Secondly, knowledge of the early time course could give insights into the reaction of the surrounding tissue and contribute to our understanding of tissue compatibility. From this point of view, the results of our study revealed interesting findings for adhesion prevention on the basis of a better understanding of adhesion formation. In addition, interesting findings regarding the peritoneal tissue reaction of this specific barrier material are evident, especially the finding that the inflammatory reaction in the treated group is not significantly higher than in the control group.

From a general viewpoint, the inflammatory response is an essential reaction after tissue damage and is crucial for the induction of wound healing [35]. In this context, a recent review by Hellebrekers and Kooistra [36] favoured the anti-inflammatory strategy in adhesion prevention. However, in the present study the inflammatory reaction of both analysed groups showed only minimal differences. Thus, in both the control and barrier samples, marked inflammation occurred at a comparable level not only immediately after tissue damage but also over the course of eight days. Regarding the granulocytic and lymphocytic migration separately, an interesting tendency towards a slight decrease of granulocytes combined with a mild increase of lymphocytes over time could be seen, reflecting the widely accepted time course of inflammatory infiltration within inflammation and wound healing. In total, the level of granulocytes and lymphocytes revealed marked similarities in both groups over the entire time period studied. Taking these findings together, on the one hand SupraSeal ${ }^{\circ}$ seems not to increase the inflammatory response, indicating good tissue compatibility of this material. On the other hand, the significant reduction of adhesion formation by SupraSeal ${ }^{\circ}$ without differences in the extent of inflammation compared to the control samples indicates that an anti-inflammatory strategy in adhesion prevention, as recommended by some authors, should be viewed with caution [36-39]. On this issue, the role of the inflammatory response in adhesion formation as well as the proper time point for any antiinflammatory strategies in case of their implementation in adhesion prevention should be investigated in further prospective experimental studies. The finding of initial inflammation in spite of reduced adhesion formation is in marked contrast to several hypotheses for innovative adhesion prevention strategies but is in line with the theory of the pathophysiology of wound healing, in which the initial inflammatory reaction is crucial to trigger further tissue reparation and regeneration [9,12,36]. Furthermore, the time scale of inflammatory cell infiltration, with rapid granulocytic infiltration followed by the migration of lymphocytes, corresponds to experimental findings $[19,20,35]$.

An important difference between barrier-treated and barrieruntreated specimens was found in the gradient of fibrin. SupraSeal'treated samples initially revealed a higher extent of fibrin compared to the control which was followed by a rapid decrease to zero within four days. In contrast, in the control specimens eight days were required to reach the zero base line for fibrin. Since fibrin plays a major role in adhesion formation, this might be one reason for the tested barrier's good preventive effect [40]. One explanation for the early disappearance of fibrin in the barrier sides could be a sealing function of SupraSeal by which the wound area including the initial fibrin clot is enclosed. Isolated in a confined place, the fibrin exudated by the wound then could rapidly be lysed due the fibrinolytic activity of the granulation response at the wound surface. However, the fate of fibrin on the polylactide-based polymer membrane still has to be clarified in further investigations. An alternative reason could be the early presence of cells with fibrinolytic capacity, mainly mesothelial cells or their precursors. This hypothesis is supported by the results of prior investigations, in which an early re-mesothelialization after the dressing of peritoneal wounds with Supra Seal ${ }^{\circ}$ was detected $[18,31,41]$. However, the results of the present study lend support to the hypothesis of the importance of rapid fibrin clearance, which is also favoured as a relevant issue in adhesion prevention by several authors $[9,11,23,36]$.

In addition to innovative aspects with a view to adhesion prevention, the present study revealed interesting information regarding the peritoneal tissue reaction of the used barrier material. In this context, it is stressed that there were no statistical differences between the inflammatory reaction to treated and untreated samples. Moreover, the cell count of granulocytes and lymphocytes revealed comparable levels in the control and treated specimens, which 
Citation: Planck CNE, Schmitt VH, Krämer B, Mamilos A, Rajab TK, et al. (2014) Histological and Immunohistological Evaluation of the Tissue Response of a New Barrier Material Based on D,L-Polylactide, Trimethylene Carbonate and Caprolactone to Prevent Peritoneal Adhesion Formation. J Tissue Sci Eng 5: 138. doi:10.4172/2157-7552.1000138

Page 8 of 9

indicates that the used barrier material did not induce a higher cellular reaction than the control group. As could be expected, the foreign body reaction at treated sites was higher than in the control samples. Taken together with the mild influx of granulocytes and lymphocytes, it becomes obvious that the material itself or possibly its degradation products trigger no further inflammatory response, which indicates good biocompatibility. Regarding the infiltration with CD68-positive macrophages, no striking difference was present between the control and the barrier group. Since different subgroups of macrophages exist with diverse, partly opposing functions, these subgroups should be assessed to give further insights in their role in wound healing and adhesion formation. In future studies, the macrophage response to the barrier material SupraSeal ${ }^{\circ}$ and its degradation products could be analyzed via in-vitro assays. Finally, the clinical and histomorphological results of the present study support the results of two former experimental studies which demonstrated good clinical results after 14 days $[8,9]$. These studies also showed that after 14 days no relevant fibrosis or scar formation occurred after treatment with SupraSeal', which could be confirmed by the present results. The present study indicates that early processes after serosal damage predict the formation of peritoneal adhesions, especially a rapid fibrin clearance which seems to be essential in adhesion prevention, whereas early inflammation would not appear to be a critical parameter for adhesion formation.

In conclusion, the present study demonstrates the efficacy of SupraSeal ${ }^{\circ}$ regarding adhesion prevention. Moreover, with the help of the time course analysis we identified rapid fibrin clearance as a potential reason for this effect. Thus, our findings show that the analysis of the time course of tissue reaction in antiadhesive strategies gives insight into the mechanisms underlying positive and negative effects of potential barrier materials.

\section{Acknowledgments}

We thank Mrs. Silke Mitschke and Mrs. Barbara Platz from the Institute of Pathology, University Medical Centre of the Johannes Gutenberg-University Mainz, Germany for their excellent technical support.

\section{References}

1. Imudia AN, Kumar S, Saed GM, Diamond MP (2008) Pathogenesis of Intra-abdominal and pelvic adhesion development. Semin Reprod Med 26: 289-297.

2. diZerega GS, Campeau JD (2001) Peritoneal repair and post-surgical adhesion formation. Hum Reprod Update 7: 547-555.

3. Brochhausen C, Schmitt VH, Rajab TK, Planck CN, Krämer B, et al. (2011) Intraperitoneal adhesions--an ongoing challenge between biomedical engineering and the life sciences. J Biomed Mater Res A 98: 143-156.

4. Weibel MA, Majno G (1973) Peritoneal adhesions and their relation to abdominal surgery. A postmortem study. Am J Surg 126: 345-353.

5. Parker MC, Wilson MS, Menzies D, Sunderland G, Thompson JN, et al. (2004) Colorectal surgery: the risk and burden of adhesion-related complications. Colorectal Dis 6: 506-511.

6. Menzies D, Ellis H (1990) Intestinal obstruction from adhesions--how big is the problem? Ann R Coll Surg Engl 72: 60-63.

7. Monk BJ, Berman ML, Montz FJ (1994) Adhesions after extensive gynecologic surgery: clinical significance, etiology, and prevention. Am J Obstet Gynecol 170: 1396-1403
8. Rajab TK, Wallwiener CW, Brochhausen C, Hierlemann H, Kraemer B, et al. (2009) Adhesion prophylaxis using a copolymer with rationally designed material properties. Surgery 145: 196-201.

9. Wallwiener M, Brucker S, Hierlemann H, Brochhausen C, Solomayer E et al. (2006) Innovative barriers for peritoneal adhesion prevention: liquid or solid? A rat uterine horn model. Fertil Steril 86: 1266-1276.

10. Dewilde RL, Trew G (2007) Postoperative abdominal adhesions and their prevention in gynaecological surgery. Expert consus position. Gynecol Surg 4:161-168.

11. Dewilde RL, Trew G (2007) Postoperative abdominal adhesions and their prevention in gynaecological surgery. Expert consensus position. Part 2steps to reduce adhesions. Gynecol Surg 4:243-253

12. Brochhausen C, Schmitt VH, Krämer B, Rajab TK, Wallwiener M et al. (2009) Intraperitoneale Adhäsionen - Eine Herausforderung an der Schnittstelle von Materialforschung und Biomedizin. BIOmaterialien 10: 7-17.

13. Arnold PB, Green CW, Foresman PA, Rodeheaver GT (2000) Evaluation of resorbable barriers for preventing surgical adhesions. Fertil Steril 73: 157-161.

14. Brochhausen C, Schmitt VH, Planck CN, Rajab TK, Hollemann D, et al. (2012) Current strategies and future perspectives for intraperitoneal adhesion prevention. J Gastrointest Surg 16: 1256-1274.

15. Glucksman DL (1966) Serosal integrity and intestinal adhesions. Surgery 60: 1009-1011

16. Hubbard TB Jr, Khan MZ, Carag VR Jr, Albites VE, Hricko GM (1967) The pathology of peritoneal repair: its relation to the formation of adhesions. Ann Surg 165: 908-916.

17. Raftery AT (1973) Regeneration of parietal and visceral peritoneum: an electron microscopical study. J Anat 115: 375-392.

18. Brochhausen C, Schmitt VH, Rajab TK, Planck CNE, Krämer B et al. (2012) Mesothelial morphology and organisation after peritoneal treatment with solid and liquid adhesion barriers--a scanning electron microscopical study. J Mater Sci Mater Med 23:1931-1939.

19. Bittinger F, Brochhausen C, Köhler H, Lehr HA, Otto M, et al. (1998) Differential expression of cell adhesion molecules in inflamed appendix: correlation with clinical stage. J Pathol 186: 422-428.

20. Brochhausen C (2002) Die Expression und Kinetik von Zelladhäsionsmolekülen in der entzündeten Appendix vermiformis: Ihre pathophysiologische und diagnostische Relevanz. Verlag Neue Wissenschaft, Frankfurt am Main.

21. Cheong YC, Laird SM, Li TC, Shelton JB, Ledger WL, et al. (2001) Peritoneal healing and adhesion formation/reformation. Hum Reprod Update 7: 556-566.

22. Liakakos T, Thomakos N, Fine PM, Dervenis C, Young RL (2001) Peritoneal adhesions: etiology, pathophysiology, and clinical significance. Recent advances in prevention and management. Dig Surg 18: 260-273.

23. Vipond MN, Whawell SA, Thompson JN, Dudley HA (1990) Peritoneal fibrinolytic activity and intra-abdominal adhesions. Lancet 335: 1120-1122.

24. Diamond MP, El-Hammady E, Munkarah A, Bieber EJ, Saed G (2005) Modulation of the expression of vascular endothelial growth factor in human fibroblasts. Fertil Steril 83: 405-409.

25. Lucas PA, Warejcka DJ, Young HE, Lee BY (1996) Formation of abdominal adhesions is inhibited by antibodies to transforming growth factor-beta1. J Surg Res 65: 135-138.

26. Schmitt VH, Brochhausen C, Planck CNE, Krämer B, Wallwiener M et al. (2009) Different barriers for the prevention of peritoneal adhesions - a histological and scanning electron microscopical study. BIOmaterialien 10:147.

27. Uhlig C, Rapp M, Hartmann B, Hierlemann H, Planck H, et al. (2007) Suprathel-an innovative, resorbable skin substitute for the treatment of burn victims. Burns 33: 221-229.

28. Kraemer B, Wallwiener M, Brochhausen C, Planck C, Hierlemann H, et al. (2010) A pilot study of laparoscopic adhesion prophylaxis after 
Citation: Planck CNE, Schmitt VH, Krämer B, Mamilos A, Rajab TK, et al. (2014) Histological and Immunohistological Evaluation of the Tissue Response of a New Barrier Material Based on D,L-Polylactide, Trimethylene Carbonate and Caprolactone to Prevent Peritoneal Adhesion Formation. J Tissue Sci Eng 5: 138. doi:10.4172/2157-7552.1000138

Page 9 of 9

myomectomy with a copolymer designed for endoscopic application. J Minim Invasive Gynecol 17: 222-227.

29. Schwarze H, Küntscher M, Uhlig C, Hierlemann H, Prantl L, et al. (2007) Suprathel, a new skin substitute, in the management of donor sites of split-thickness skin grafts: results of a clinical study. Burns 33: 850-854.

30. Schwarze H, Küntscher M, Uhlig C, Hierlemann H, Prantl L, et al. (2008) Suprathel, a new skin substitute, in the management of partial-thickness burn wounds: results of a clinical study. Ann Plast Surg 60: 181-185.

31. Brochhausen C, Schmitt VH, Wiedenroth CB, Krämer B, Rajab TK, et al. (2008) Remesothelialization of a polylactide-based membrane for the prevention of peritoneal adhesions. Eur Cell Mat 16:26.

32. Rajab TK, Wauschkuhn CA, Smaxwil L, Kraemer B, Wallwiener M, et al. (2010) An improved model for the induction of experimental adhesions. J Invest Surg 23: 35-39.

33. Wallwiener CW, Kraemer B, Wallwiener M, Brochhausen C, Isaacson $\mathrm{KB}$, et al. (2010) The extent of adhesion induction through electrocoagulation and suturing in an experimental rat study. Fertil Steril 93: 1040-1044.

34. Kraemer B, Rothmund R, Fischer K, Scharpf M, Fend F et al. (2011) A prospective, randomized, experimental study to investigate the peritoneal adhesion formation of noncontact argon plasma coagulation in a rat model. Fertil Steril 95:1328-1332.

35. Li J, Chen J, Kirsner R (2007) Pathophysiology of acute wound healing Clin Dermatol 25: 9-18.
36. Hellebrekers BW1, Kooistra T (2011) Pathogenesis of postoperative adhesion formation. Br J Surg 98: 1503-1516.

37. Binda MM, Molinas CR, Bastidas A, Koninckx PR (2007) Effect of reactive oxygen species scavengers, antiinflammatory drugs, and calcium-channel blockers on carbon dioxide pneumoperitoneumenhanced adhesions in a laparoscopic mouse model. Surg Endosc 21:1826-1834.

38. Oh SH, Kim JK, Song KS, Noh SM, Ghil SH, et al. (2005) Prevention of postsurgical tissue adhesion by anti-inflammatory drug-loaded pluronic mixtures with sol-gel transition behavior. J Biomed Mater Res A 72: 306-316.

39. Suckow MA, Hodde JP, Wolter WR, Wood KV, Hiles MC, et al. (2010) Addition of nimesulide to small intestinal submucosa biomaterial inhibits postsurgical adhesiogenesis in rats. J Biomed Mater Res B Appl Biomater 93: 18-23.

40. Arung W, Meurisse M, Detry O (2011) Pathophysiology and prevention of postoperative peritoneal adhesions. World J Gastroenterol 17: 4545-4553.

41. Brochhausen C, Krämer B, Rajab TK, Schmitt VH, Wallwiener M, et al. (2007) First results of the remesothelialization of a polylactide based foil for peritoneal adhesion. BIOmaterialien 8:150. 